
\title{
Discursive Practices in Cooperative Structures
}

\author{
Nikolay Viktorovich Vedin“, Valery Vladimirovich Shlychkov and Asya Shamilevna Khasanova \\ Department of Economic Theory, A. N. Tupolev Kazan National Research Technical \\ University - KAl, Kazan - 420111, Russia; nick_vedin@mail.ru
}

\begin{abstract}
The article reveals a system-forming role of verbal communications in the economic organization of society, based on cooperative relationships. The authors identify the limits in the use of individualistic methodology in the problems of economic organizations. The approach to organization analysis is implemented in the light of contradictory unity of cooperation and competition. In the context of delimiting the functional and discursive domains of collective production, the article brings to light the nature of basic institutional forms which regulates production and exchange in the public economy.
\end{abstract}

Keywords: Competition, Cooperation, Discourse, Exchange, Institutions

\section{Introduction}

The twentieth century has considerably changed the theoreticians' views. The strengthening of corporate trends in the economies of the developed countries, the increased attention to intracorporate management, corporate culture, human assets and organizational structures and company competitiveness gradually made adjustments to the market model. The economists, both theoreticians and practitioners, believe that the market sphere is getting more densely populated by formations, inside which nonprice and even non-economic instruments of commercial success were widely used.

The progressive extension of the social context of the modern economic science is a natural process which draws the economists' attention to knowledge, skills, culture, education, innovation, trust and cooperation norms ${ }^{1}$. At the same time, the economic theory has only partially risen to the socio-humanitarian challenge, using approaches and tools of the economic worldview that was formed in the preceding historical period for a selective analysis of the new phenomena. In particular, it was reflected in the theories of human and social capital. Meanwhile, the selective and purely instrumental market-based approach to these elements conceals their systemic unity rather than reveals it. Despite their functional diversity, they all are the products of the same language-discursive domain of cooperation. This research represents an experience of analyzing and justifying the specific economic nature of this domain, which results in recognition of the heterogeneity of the economic organization of society, defined as a contradictory unity of cooperation and competition.

\section{Methodology}

Methodological basis of this work comprises the tools of dialectical logic, i.e. dialectic of the logical and historical, general and specific, the logic of contradiction, as well as the dialectically meaningful methods of analysis and synthesis, induction and deduction. The distinctive feature of this study, based on the systematic approach, is the application of methodological holism and its specifictheoretical interpretation as a basic model of cooperation which has a real empirical referent. Along with the systemic principle, the following general scientific tools are used in the study: The hypothetical method and abstraction, the synergetic approach, the comparative analysis, the methods of heuristic modeling and extrapolation.

\footnotetext{
${ }^{*}$ Author for correspondence
} 


\section{Results}

\subsection{Economic Organization: The Problem of Alignment of Competitive and Co- operative Relations}

The desire to understand the nature and consequences of the real expansion of non-market forms of human communication, based on values which do not lend themselves to commercial calculation, resulted in a significant extension of the problematics in the economic theory. On the one hand, science, at least its mainstream, demonstrates a kind of an extensive path of development, applying the traditional mainstream instruments (methodological individualism, rational choice, equilibrium theory) for explaining human behavior in such spheres as family, marriage, crime, education, religion and sexual relationships which are "sensitive" for the economic theory. On the other hand, such universalist expansion of the market orthodoxy possibly played its role in the formation of an intensive path of economic science development, stimulating the search for alternative approaches to the analysis of human relationships at the micro and macro levels of economic reality.

This movement is clearly found in the works of agreement economy representatives, in particular, in their reference to the role of language in the institutional environment, interpretational rationality, incompatible with the market exchange and in their search for procedures of the identifying a common interest, which does not find its place in the standard theory ${ }^{2}$. The interpretation of the company as a non-market "institution of knowledge integration", the increased interest in learning issues and the evolution of the workers' abilities in terms of a "resource-based" approach ${ }^{3}$ demonstrate the diversion from mainstream thinking and an attempt to contemplate the economic reality in a new light.

Positively assessing these approaches and the ones close to them, implemented, in particular, by evolutionists, we qualify them, according to V. Leontiev ${ }^{4}$, as an iterative movement towards understanding cooperation as the fundamental basis of the socio-economic reality. The fact that civilization is generally based on cooperation as a fundamental norm of social life is proved not only by the mankind experience in its broad historical retrospect, dating back to the archaic community forms. In the long run, all human daily life consists of various forms of co-operation with other people. Even Alfred Marshall pointed out that the factors encouraging people to compete with each other, at the same time "push people towards establishing co-operation and creating all kinds of associations, both selfish and unselfish" .

However, the reference to the classics' experience and evidence only serves to indicate a problem. The latter consists in the necessity of a theoretical reconstruction of a real symbiosis and intertwinement of co-operation and competition relationships. All attempts to find in this symbiosis any traces of collaboration, whether it is a dependent individual, common interest, non-market coordination, etc., contradict their involvement in overall competitive market environment. Apart from this, conceptually, in a scientific explorer's non-reflected commitment to a particular tradition, they are integrated into the competitive market worldview, since there exists no other one. It makes even the opponents of market orthodoxy adjust the fragments of co-operation to the market realities at the cost of ignoring their systemic nature among other ways.

Economists' turning to organizational analysis resulted in thefact that the study encountered a previously unknown problem of combined economic systems (forms) of labor division and activity exchange i.e. competitive market and cooperative form of co-operation. Combined in reality, in the form of an unobserved duplication of economic existence of the economic process participants, they can be analyzed only under condition of their preliminary theoretical splitting (mutual abstraction) with their subsequent theoretical reintegration into a new integrity. It can be assumed that in this integrity, new properties, which will prompt the solution of still unsettled tasks, will be discovered ${ }^{6}$. To such tasks we can refer, for example, searching for ways to neutralize opportunistic behavior of organization members or the problem of measuring the peak productivity of individual workers in order to determine their contribution and adequate remuneration.

A. Alchian and G. Demsetz ${ }^{7}$. as it seems to us, managed to reveal the essence of the problems, which occur when dealing with such tasks. They realize that the final product of collective production is not a simple combination of separate outputs which are identical in terms of the corresponding parameters. This is reflected in the inseparability of the production function. Hence, "what the team offers the market can be used to measure the marginal product of the team, but not its members". 
But without measuring an individual marginal product, it is impossible to accurately determine the remuneration and thereby neutralize the supposedly natural desire of individuals to shift the work to others and completely edge away from their duties. The complexity of measuring engenders a problem of monitoring and control, the cost of which in their turn, hampers their totality and hence their effectiveness.

Naturally, there are various options of at least alleviating the problem if not solving it. Some of these options are dealt with in the article written by Alchian and Demsetz. However, we are concerned with another aspect of this problem. In fact, we are facing a system where only one person is interested in the efficient and conscientious work of all the others. This person is the owner of the company. However, he withstands not only the employees, but also the owners of another type of resource - human capital. Thus "collective production" appears to be an association of private owners, among whom it is appropriate to bargain aiming to gain a "share" advantage. You have to be very indulgent to consider such association to be a team. Theoretically, it is impossible to expect them to do their work effectively and in a wellcoordinated manner.

In practice such pseudo-teams are very rare, being torn apart by the selfishness of private interests. Taking the competitiveness criterion as a basis we will undoubtedly see the leaders and the ones lagging behind. This is a fact, as well as the fact that organizations quite often swap places in this race. The fact of the matter is not only and not so much in the managers' talents, as it appears from the managerial interpretation of the firm. If it (interpretation) is given a logically completed form, the competitiveness of the company turns out to be the result of voluntary, whether talented or ungifted, loyal or opportunistic, actions of the manager, who chooses an appropriate strategy and organizes its implementation taking into account the internal and external business environment. The guarantee of success is the manager's talent and their personal motivation matching the target function of the organization. In practice, there have been such examples, although both conditions are fairly unique. Hardly anyone will deny that a talented and honest administrator is a rare phenomenon. In any case, there are far fewer talents in this sphere than there are successful and competitive firms, like in any other sphere.

It would be rash to deny, to one extent or another, the existence of motivational disunity within the members of organizations. Such disunity exists, as long as competitive market relations based on private property and contracts of employment are a reality. But, first, it is not absolute and secondly, it can create positive work incentives in certain circumstances. By certain circumstances we mean not only the efficient organization of production, but the existence in production teams of a powerful centripetal factor, which represents the greatest interest in the context of this study. It can be assumed that a deep layer of economic relations that give genuine collectivity to "collective production" hides itself under the marketing cover of private interests and opportunistic manifestations. This refers to the relations of $\mathrm{CO}^{-}$ operation. The fancy interweaving of co-operation and competition forms gives rise to a great variety of strategic preferences and business success not only of commercial organizations, but also of national economies. The sociocultural environment of various national economies has a specific and unique (for each ethnic group) impact on the economic sphere specifically through a system of cooperation.

\subsection{The Clash of the Individualistic and Holistic Approaches to Organizational Analysis: The Formation of Research Preferences}

Running slightly ahead, we shall single out only two main factors: The reality of common interest and discursive (verbal) form of activity exchange, which lend the specificity to the system and precondition its synergy. The analysis of the corresponding structure of relations based on these premises is possible solely in case of preliminary abstraction from commodity (competitive market) form. The dramatics of the situation lies in the fact that the procedure of splitting and synthesis of competition and cooperation entails many problems, both of methodological and purely theoretical character. In particular, the problem of methodological individualism, which turned into a peculiar research tradition long ago despite the fairly active criticism, is being aggravated. This principle is not even reflected on by many researchers, but is used as a usual way of reasoning. Meanwhile, universalizing the individualism hinders understanding the nature of co-operation, for which it is natural to use the approach from the perspective of integrity. With a certain degree 
of probability we can anticipate three main scenarios that characterize the further formation of possible exploratory preferences and research directions in this area.

First, remaining within this ambiguous cognitive situation, some researchers will inevitably drift towards a more well-founded and logically constructed theory which market orthodoxy represents. An example of this is the destiny of the institutional approach and its most influential direction - neoinstitutionalism.

Secondly, some economists who are unwilling to drift in this direction, but not being able to find the means to get rid of the pressure of the intellectual mainstream, which is supported by the market empirism, will continue to accumulate and classify the facts characterizing the failure of individualistic methodology and "organizational uniqueness". These efforts can only be welcomed.

And, finally, the formation of some of the above mentioned modern trends, opposing the market orthodoxy, serves as evidence of the fact that their representatives are considerably close to understanding the need for a consistent, conceptually shaped abstraction (at a certain stage of study) from the competitive market environment. It should be pointed out that justification for such abstraction is the fact that the form of co-operation is feasible as such in its "pure form", beyond competitive market inclusions. Competition and market historically grow out of co-operation and are impossible beyond the co-operation. The fact that in modern economy the form of co-operation goes into the base of economic system, turning into an unobservable (at least, for the market orthodoxy) economic reality, does not make any fundamental changes to the problem statement ${ }^{8}$.

\subsection{The Basic Model of Co-operation}

The form of co-operation directly corresponds to the notion of co-operation. In the economic theory, this concept is not strictly formulated. Thus, there has been a recording of interpreting the market organization as a result of market co-operation' ${ }^{9}$. Based on the functionalbranch characteristic this concept is used to describe all sorts of local organizations i.e. industrial, consumer, sales, credit and housing co-operation. Generally, taking into consideration the interdependence of co-operation and collaboration concepts, we can notice that they are used to refer to a wide range of mutually beneficial public relations, including the commercial sphere, political, scientific and technical relations, culture, etc. A certain vagueness of the term "co-operation" does not prevent its sufficiently wide use by economists, which testifies to its theoretical relevance.

Classics of political economy applied it in different contexts and with a varying degree of certainty, but mostly on the periphery of their conceptual compositions. Perhaps, the greatest interest in co-operation was shown by Karl Marx, who described it as a form of labor, in which many individuals systematically works alongside and in collaboration with each other in the same production process or in different but related processes of production ${ }^{10}$.

An idea of collective production as such, as a form of collective work, is based on two premises. Firstly, this form should not be treated as the result of either separate deals between owners of heterogeneous resources, whether they are contracts of employment or profit-sharing contracts or any transactions that can bring into the organization the interests which are external to the collective labor itself. The form of collective labor exists in the researcher' mind as a reality, the existence of which is confirmed by the experience of all the previous generations.

Secondly, the form of collective labor owes its specific nature to the typological unity of functional labor division and real activity exchange. In this case the functionality is not a technical characteristic of fragmentary activity (for example, in the modern assembly industry), but an economic category. It conveys the dependence, partiality of individual actions of each employee in terms of creating the social product. Even if this product has a complex structure, as is the case with a patriarchal household, for example, it is manufactured only by collective efforts of many workers, each performing a specific function. Consequently, in collective production there are no prerequisites for exchange of products as commodities and the exchange itself, if necessary, is represented by the movement of knowledge and skills and takes the form of verbal communication (discourse). Thus, co-operation presupposes the production community or an aggregate worker on the one hand and their social product on the other hand. The proportions of individuals' participation in the consumption of the social product are preliminarily set and identified by objective conditions and interests of this community.

\subsection{A Dependent Individual and Verbal Communication in Collective Production}

An individual's participation in the collective production 
therefore lies in the fact that theyinvest their share of livinglabor into the collective activity. Thus, in its external definiteness a worker's partial labor represents simple and single human energy consumption in a functional form. Such labor can only be measured by actual working time, because the individual themselves operates at all points in the production process as an integral part of the aggregate worker.

However, equal amounts of individual time cannot but differ from each other by the content of their live activity, taking into account the differences in skills, knowledge and physical strength of workers, which cannot but impede achieving an overall result. This circumstance is most evidently manifested in developed forms of the collective labor, where any working function or manufactured product at any given moment is mediated by the activity of many individuals i.e. by public interaction. Taken any method of combining individual efforts, every employee affects the subject of labor in a manner which is conditioned by the total of public actions with it. The question is not only in the technology of actions but also in the intensity of labor and the quality of performing a particular action.

So the actual working hours can represent a publicly recognized measure of labor only to a certain extent as in the production process there is the alignment of individual skills and labor costs which are brought to the level, appropriate for these conditions, through peer learning, exchange of experience, discussing production problems, i.e. various forms of verbal communication or discursive practices.

Speech or language communication is defined by Y. Habermas as "communicative mind", which in contrast to the "instrumental", functionally oriented mind, is aimed at understanding between people ${ }^{11}$. As language communication, discourse presupposes a synchronized process of objectification and subjectification of content in human consciousness. By perceiving and subjectifying information codes, a member of discourse voluntarily or involuntarily makes the "another person's" reflections and abilities his "own" and, vice versa, through informationlanguage encoding, makes their thoughts and abilities available for perception and conceptual interpretation by other members of communication. A lack or destruction of communicative mind makes it impossible not only to co-operate in teamwork, but to do mutually beneficial activities in marketing. It blocks the constructive effects of competition, turning it into a war of all against all.

The concept of discursive practices which this article is devoted to, presupposes the existence of varieties of discourse, which may differ from each other by procedures, regulations, the type of rationality and targets. In subject boundaries of the economic theory, economic discursive practices are treated as a specific way of exchanging and integration of individuals' active abilities in the collective production. The notion of discursive practices presupposing social dependence of verbal communication, more accurately reflects the essence of co-operation compared to the broader notion of information exchange, which focuses on the movement of objectified knowledge, storage media (including marketbased ones). Strictly speaking, information exchange can take place also in technical systems or in the animal world. Discourse, however, differs by focusing on deeply personal, unalienated interaction processes in collective production. It is in the domain of verbal communication that the social but always personal contradiction between a human desire for independence and the equal striving to be involved in a group is solved and reproduced. So, the possibility of an individual's (or a group of individuals) isolated business activity under certain conditions lies in the discourse as such. This is important for understanding the origin of commercial manufacturing.

According to the economic theory, coordinating the activities of many individuals, acting as private producers or participating in the same production process, is always an objective socio-economic connection, integrating individual efforts into achieving a social result. And the main essence of this connection is the exchange of living (co-operation) and materialized (commodity form) activity.

How appropriate is it to identify this economic process with planning or, moreover, with management? Despite all the specificity and significance of this type of activity, it represents only one of the functions of collaborative labor and it would be a mistake to treat it as an instrument of social recognition of all other individual costs or as a sort of supreme public authority. We would then have to acknowledge the existence of such function, which, in its turn, would "authorize" a public nature of managerial work, etc.

Note that the interpretation of the immediate social labor in Marxist political economy on the basis of the planned feature which ensured public recognition of individual labor costs long before the production process subsequently initiated a problem of incompatibility of the plan and the market in socialism ${ }^{12}$. 
From the point of view of common sense, in order to explain the causal relationship between each employee's partial work and the social product (social usefulness) as a result of the collective labor, it is enough to state the fact of seeing people working together hand in hand. But this means that under the conditions of co-operation the economic link between different types of work, i.e. exchange, are provided by the manufacturing process itself. Consequently, the exchange of activity is its (the production process) internal moment and the subject of the exchange where appropriate does not take the form which is different from the living activity. If we take into account the fact that work activity is the realization of a person's productive abilities, then it is them who are the real subject of the exchange. Only such exchange can provide the alignment of the individual work force and, consequently, the public recognition of individual costs in the process of collaborative labor.

\subsection{Productive Exchange in the System of Co-operation}

Despite the unconventionality of the discourse problematics for the economic theory, there have already been attempts in the literature if not at its particular development, but at least, at formulating a problem and finding the ways to solve it. Apart from this, in terms of the evolutionary and resource approaches to organizational analysis, certain work has been done to clarify the structure issues, the formation and the use of company employees' knowledge and skills. The conceptual basis of these developments is constituted mainly by $\mathrm{M}$. Polanyi' ${ }^{13}$ ideas concerning the existence of explicit and implicit (non-articulated) knowledge. This idea is based on the statement of the fact that "a person knows more than they can say". In other words, the desired result of the action "is achieved by way of following a series of rules or regulations, unknown as such to a person who performs this action".

The economists using M. Polanyis idea in their developments, place the main emphasis on this implicit knowledge and its deep personal nature, which allows the supporters of the resource approach to discuss the issues of the resource uniqueness (specificity) as the basis for the company's competitiveness; it also allows evolutionists to discuss the issues of unpredictability of workers' actions leading to unpredictable evolutionary changes in the organization.

These studies made a very significant contribution to the development of the subjective aspect of the functioning of the organization and its employees. One might expect that the analysis of the abilities of individual workers as functional performers will be complemented by the study of their interaction (exchange) system at the level of the whole organization. But this problem, apparently, has not been raised by researchers. Regardless of the reasons for such indifference to the problem of living exchange, we should mention a common drawback inherent in works of this kind. They do not initially draw an important distinction between discursive and functional domains of co-operation. The emphasis is placed on the functional side of the workers' activity, reflecting their performing isolation, individuality, interaction with the material conditions of production. Much less attention is paid to the central problem of co-operation i.e. the actual discursive domain.

Meanwhile, it is the presence of discursive practices that makes a person's special economic "modelling" absolutely unnecessary. The meaning of an individual's "non-autonomy" in the co-operative structure is that all the economically significant parameters of their production activity such as knowledge and skills, interests and goals, degree of awareness, type of rationality, are formed by the conditions of their integration into the structure of the general economic whole. The only prerequisite for an individual's integration is their intelligence and ability to learn, which is equal to the subject's recognition as having the homo sapiens status.

Whatever explains the difference in the productive capacity of co-production participants - a person's physiological characteristics, different work experience or its absence altogether, level of education, individual aptitude, etc. - it exists at any given moment of the company functioning and engenders the problem of agreed (coordinated) performance of interrelated and complementary functions of the collective labor. In this essential point our position diverges from the interpretation of "a typical member of organization", which is offered by R. Nelson and S. Winter ${ }^{14}$. For the authors of the evolutionary theory "a typical member of organization", equipped with the corresponding individual skills and routines, is an accomplished prerequisite for analyzing organizational dynamics.

At first glance, such approach looks realistic, since it corresponds to the empirical static of the functioning organization and its "snapshot" on which an observer can see a lot of people, each doing their activity equally 
competently. But from the point of view of organizational dynamics this static approach greatly reduces the possibility of detecting changes at an individual's level and consequently, at the organization level. In fact, "a typical member of organization" is an abstraction that has little in common with the actual production participants, who, to a certain degree, not only possess the routines they are supposed to have according to the "repertoire", but may not have any special training whatsoever.

It is important to emphasize that the relationship between separate fragments of the production process potentially exists beyond the workers themselves, in real production conditions (e.g. in the system of machinery). It is this functioning technical system, as if standing in front of the researcher's eyes, that gives an impression of an organization's ordered well-being. However, the starting point in understanding the structure of collective production lies not in this technical (or natural) connection between individual elements of production, but in the individual specific features of performing them by separate employees. These features can show a different degree of approximation to the optimal version of how the production system can function as a whole and as separate elements. But at any rate, the participants of the production process must function as a single production force, in which the abilities and actions of some subjects are agreed with those of the others.

Such agreement, at the first approximation, implies mutual correction of the sequence, the method of implementation, the speed of operations, i.e. skills. By skills we mean an individual's ability to perform certain operations (actions) with the specified parameters (sequence, intensity, methods) which presuppose achieving a certain goal. But the mutual correction of individual skills is possible only if they are "transparent", available to all members of the organization. If Polanyi's "implicit knowledge" is present here, then we either find a way of practically transferring it into the category of explicit knowledge or theoretically it might not be taken into account in the context of discourse analysis. The transparency of individual skills for collaborating subjects can be interpreted as their desire for mutual understanding of each other's actions. But if an individual "understands" another subject's way of behavior, it means that they make another person's way of behavior their own one, although it does not imply the action of copying. And as individuals constantly swap their roles in these processes, the process of mutual understanding in the collective production takes the form of a living activity exchange, i.e. the exchange of functioning abilities.

In the course of analysis we should take into account the fact that the activity capacities literally and physically are inseparable from a person. But at the same time they are structurally organized. Without going into phsychophysiological nuances of this issue, we shall only note that in the context of this study, by a person's creative abilities we mean, on the one hand, a contradictory unity of profound individual qualities that determine the degree of a subject's mastering a certain activity and the generally significant forms of their expression and transmission, on the other hand.

This other side is represented, above all, by linguistic means of communication between people. Being expressed in linguistic or non-verbal forms (gestures, facial expressions), human abilities lose their individuality and objectify themselves, but at the same time they acquire universality and interpersonal mobility. In their turn, linguistic forms that are perceived and interpreted by a person lose their universality, but are transformed into individual properties (creative abilities) of a personality and become a productive force. Thus, in the movement of linguistic (and non-verbal) forms, two differently directed "vectors" of personality are expressed. One has an outward direction (to the others), the other one is directed inwards (towards a person). So, discourse is predetermined by the needs of collective labor, the need to align the abilities and mutual co-ordination of individual work efforts, bringing them in line with the requirements of the specific interaction standards, etc.

Owing to the language, the workers' productive characteristics (knowledge, abilities and skills) acquire corporate mobility. This movement comes down to a permanent mutual transition of personal and objectified knowledge. The process of accumulation and development of abilities happens at the "points" of this mutual transition, where the individual interpretation and broadcasting of circulating information take place. Externally it can be found in the growth of labor efficiency, emergence of new ideas, relevant developments and implementations, acceleration of the organizational change processes. It is these processes of multiplying the creative abilities of an individual and the aggregate worker as a whole in conjunction with the competitive trends that form the basis of the so-called synergistic effect of co-operation. With this understanding, the distinction between the codified "knowing that" and 
the personal "knowing how" in terms of priority and specificity of the latter, loses its sense considerably, since these components are not only interdependent, but they are also in a constant process of mutual transition. The freedom and intensity with which the codified knowledge moves within an organization, all other conditions being equal, ensures the accumulation of its dynamic potential. At the same time, we must remember that the same processes may threaten the integrity of an organization. If they led to the disintegration of communities and the emergence of commercial production in the early stages of the co-operation genesis, in a modern organization a spontaneous accumulation and an uneven distribution of knowledge can cause an increase in co-ordination costs and the strengthening of centrifugal corporate tendencies.

"The subject" of exchange in an organization is not used in the usual sense of this word, but it is not copied either, not repeated in a mirror-like manner at any points of its movement. Labor practices and working methods are always reproduced by an employee who adopts them as their own abilities, with their usual individual originality that makes these creative elements a potentially new subject of exchange. Moreover, individual personal basis is preserved within them in a "removed manner", namely in the form of pro-innovative components i.e. original informational "additions".

The collective labor process presupposes not only a mutual "simulation of actions", but also a mutual change, an individual interpretation of methods and techniques of work. It is another matter that this interpretation does not always assume the final shape of the new method or the new skill, i.e. innovation, which denies the existing routine. However, it is from this source that the elements of new knowledge, skills, methods and structures of activity emerge.

\subsection{Institutionalization of Co-operation}

By institutionalization we understand the process of emergence, testing and regular recurrence of various socio-economic practices for a considerably long period of time. Changes of co-operation conditions lead to a change (evolution) of practices themselves and, consequently, to their relatively stable forms.

The conditions of the traditional production process, in which partial workers act as functionally separate elements of the technological system - animated working tools - limit the ability of communication. A partial employee's efforts are directed not towards other workers, but towards the action they perform, to the subject of labor. With regard to the developed forms of collective labor in machine production, a routinely performed activity, as a rule, is not reflected on by a worker and is carried out to a considerable extent unconsciously and automatically. And in this respect we cannot but acknowledge the heuristic value of Polanyi's concept of implicit knowledge.

However, it does not follow from this that a worker (performer of work) is not capable of comprehending their actions and of transferring the implicit knowledge into the category of explicit, articulated one, lending itself to transmission and exchange. But in most cases this takes time, free from performance of duties. A worker is engaged in the labor process so much that the very possibility of parallel communication with other individuals is essentially limited by the cost of switching their activity from the subject of work to a person.

It is significant that modern trends in the change of labor content and vocational and qualification structure of the personnel, contribute to overcoming and more precisely, to mitigating the specified limitation. As is shown by the results of the surveys among the members of autonomous (integrated) teams, the workers most often indicated such aspects of group work, which satisfy them and facilitate personal development as solidarity, the opportunity of making of contacts at work and a constant dialogue with colleagues, co-operation with other departments and agencies, workplace rotation and in general, the extension of social links in the production process ${ }^{15}$.

However, it should be taken into account that the realization of the "predisposition to a dialogue", the extension of "the opportunity of making contacts at work", an increasing degree of solidarity and system of participation, by themselves, do not mean a complete alignment of "parallel" social labor domains i.e. functional and discursive. Their full alignment, in all likelihood, is not achievable at all, because the work activity has always been and will be carried out in the subject-oriented form, whether it is the creation of an industrial product, software or the study of any natural or social processes.

Even scientific work, which is based on the predecessors and contemporaries' co-operation mediated by the culture and social memory, is not free from the elements of specialization and functional isolation. But if the researcher's dialogue with his opponents and supporters from the past and present is a vividly expressed 
dominant element in the scientific work, then in usual industrial practice of collective labor the tangible power of the functional component dominates the structure of co-operation so considerably that the participants and especially managers see the collective exchange (communication) as an element of excessive luxury which only interferes with the normal production process.

The contradiction between the partial functionally separated activity and the objective need for collaborative labor in the exchange of living activity is settled by the institution of consultation. It is one of the most ancient institutions governing the production and exchange processes in the system of co-operation and acting as a sustainable formation, compensating the functional limitations of the collective exchange and extending the time limits of the discursive domain of the collective labor.

Specific standards characterizing this institution can be divided into two large groups of meetings: Organized and spontaneous. Organized meetings are connected with the intragroup hierarchy; they are characterized by consistency, purpose-oriented character and ordered statements. These are various kinds of industrial assemblies, meetings, planning meetings, briefings, conducted with different degrees of regularity and procedural order. The total of various organized meetings can be described as an organizational protocol.

The organizational protocol permits structuring the co-operation domain, by procedurally separating the discursive and functional spheres. This ensures saving time which is connected with the maintenance of normal production rhythm, as well as the possibility of concentrating the information exchange (a version of "brainstorming") and the development of effective coordinated solutions without having to carry out separate interactions with the individual members of the collective production. In terms of the organizational protocol such norms of co-operation as planning and control are formed.

To spontaneous meetings we can refer occasional contacts between employees of all categories during the working day, occurring during lunch breaks, additional breaks for rest; events connected with non-standard work situations, breakdown of equipment, discussing the nuances of the new manufacturing tasks, conflicts between members of staff, etc.

E. Mayo, one of the founders of the school of human relations in the theory and practice of management, was the first to pay attention to this group of meetings and their role in the team cohesion and production efficiency. Spontaneous meetings between workers during long and short breaks as well as specially initiated communication through any organizational or technical innovations changing the conditions of collective labor intensified intra-group communication, contributing to the improvement of staff cohesion, interest in work and overall productivity growth.

The ratio between the institutional protocol and spontaneous meetings in the history varied because of their fusion, when more or less distinct boundaries between them did not exist whatsoever (which is characteristic of archaic economic forms in which the company was absorbed by the elements), through the period of industrial practice which contributed to the displacement of spontaneous meetings by organized ones and to the modern innovation-oriented, creative organizations, where spontaneous meetings prevail at the same time possessing separate traits of being organized. We can state that neither of these types of meetings can be eliminated, but the boundaries between them are changing depending on historical conditions in the broad evolutionary context, as well as on the strategic preferences of certain organizations (firms) in the modern market economy.

\section{Conclusion}

The reality of the co-operation relations, as well as the competitive market forms, is confirmed by all the historical practice of the functioning and development of the economic systems. The researchers' attempts to present non-commodity (immediately social, plannedprescriptive) production as a system "non-exchange" based on a centralized, team authorization of the public value of the manufacturers' labor (whether it is a company employee or a comparatively isolated enterprise in the planned economy), gave rise to the problem of incompatibility of these forms. The analysis of the economic structure of collective production, based on the exchange of living activity (verbal communication), allows us to understand the ratio of these forms as an economic symbiosis of different but compatible ways of coordinating economic behavior of the same participants simultaneously involved in different exchange systems with different basic characteristics of the subject, motivations, methods and results of activity exchange. 
This creates an essential possibility of forming the modern economic worldview which, unlike the onedimensional and competitive market model, is based on the heterogeneity of economic systems.

This article does not cover a number of important issues related to the nature and specific features of realizing collective and personal interest, the economic structure of working hours in the conditions of collective production, the essence of organizational control, the role of traditions and innovations in the functioning and development of co-operation system, etc. The above, however, is sufficient to conceive the value of discursive practices in collective production. At the same time, it should be noted that the "micro-level" of the conducted analysis should not mislead the reader concerning the significance of its results only for the national economy; they are also significant within the boundaries of the global economy. As Pierre Teilhard de Chardin would remark in this connection, "a nucleus of the universe is the universe itself" ${ }^{\prime \prime}$.

\section{References}

1. Khasanova ASh,Vedin NV. Formation of new economic paradigm. The Review of Economy, Law and Sociology. 2014; 4:106-11.

2. Teveno L. Values, coordination and rationality: Economics of agreements or the epoch of economic, social and political sciences rapprochement. Institutional economics: Textbook. Moscow: INFRA-M. Nauka; 2007. [in Russian].

3. Luksha P, Belousenko M. Economic organization: On the way to synthetic theory. Voprosyekonomiki. 2006; 2:99115. [in Russian].

4. Leontyev V. Economic essays. Theories, investigations, facts and politics. Moscow: Mysl; 1990.

5. Marshall A. Principles of the economic science. In 3 vols. Moscow: "Progress". 1993; 1. [in Russian].
6. Shlychkov VV. 400 years of evolution: From political economy to "Economics". The Review of Economy, Law and Sociology. 2014; 4:112-4. [in Russian].

7. Alchian A, Demsetz H. Production, information costs and economic organization. American Economic Review. 1972; 62:777-95.

8. Shlychkov VV, Nestulayeva DR. Economic science and evolution of economic agents' expectations formation process. Actual Problems of Economics and Law. 2015; 3:5-16. [in Russian].

9. Furubotn EG, Richter P. Institutions and economic theory: Achievements of new institutional economic theory [Tr. from Eng.]. St. Petersburg: SPb State University Press; 2005.

10. Marx K. Capital. Critics of political economy. Moscow: Politizdat. 1978; 1. [in Russian].

11. Monson P. Modern western sociology: Theories, traditions, perspectives [Transl. from Swedish]. Saint Petersburg: "Notabene" Publishing House; 1992. [in Russian].

12. Vedin NV. Economic heterogeneity of exchange in economic evolution of the society. Saint Petersburg: NPK "ROST" Publishing House; 2006. [in Russian].

13. Polanyi M. Personal knowledge. Moscow: Progress; 1985. [in Russian].

14. Nelson PP, Winter SG. The evolution theory of economic changes. [Tr. from English]. Moscow: Delo; 2002. [in Russian].

15. Vilkhovchenko ED. Social-professional development of people in advanced countries manufacture. Mirovayaeconomika I mezhdunaronniyeotnosheniya. 1997; 8-9:85110. [in Russian].

16. Teilhard de Chardin P. The human phenomenon. Moscow: Nauka; 1987. [in Russian].

17. Vedin. A detailed analysis of the problems of the economic heterogeneity of the society's economic organization and its reflection in the political economy of socialism is contained in the study of one of the article authors; 2006. 